\section{Approach to Treatment of a Non-functional Retrovesical Bladder Paraganglioma}

Sir,

Paragangliomas are rare neuroendocrine tumors originating from chromaffin cells on the migration route of neural cells. We aim to present a case of retrovesical bladder paraganglioma, which was of non-functional type, and to discuss its treatment, by excision using laparoscopic partial cystectomy used with synchronoustransurethral imaging.

A 32-year male was admitted to our clinic with a complaint of suprapubic pain. Physical examination, routine blood tests, and urinary analysis of the patient did not reveal any pathology. There was no history of irritative voiding symptoms, hematuria, palpitations, facial flushes, or hypertensive attacks. The blood pressure (BP) was $124 / 80 \mathrm{~mm} \mathrm{Hg}$. The 24-hour urine metanephrine level was $78.8 \mu \mathrm{g}$ (reference: $30-350 \mu \mathrm{g} / 24$ hour), and normetanephrine level, $358.5 \mu \mathrm{g}$ (reference: 111-419 $\mu \mathrm{g} / 24$ hour). Ultrasonography and computed tomography revealed a hypo-echoic solid lesion of $35 \times 18 \mathrm{~mm}$, situated on the left posterolateral wall of the bladder. Its centre had a slightly hyper-echoic appearance (Figure 1).

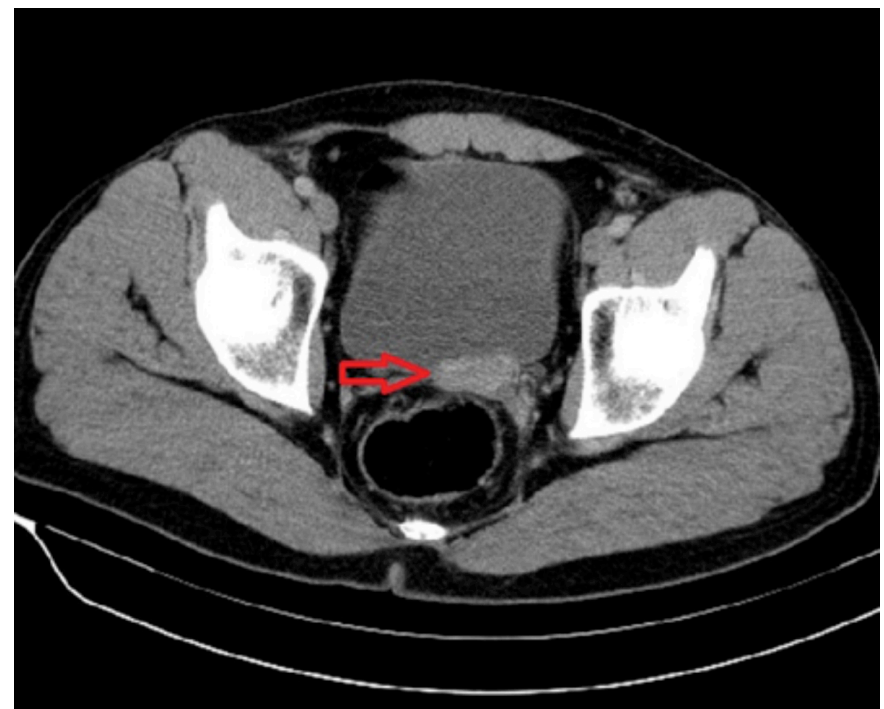

Figure 1: Retrovesical mass on computed tomography.

In the cystoscopy performed under general anesthesia, a $2 \times 3$ $\mathrm{cm}$ lesion pressing on the lumen at the opposite wall of the bladderwas observed. The biopsy result obtained from the retrovesical mass using transrectal ultrasonography was reported as neuroendocrine tumor suspicious for malignancy. On immunohistochemical examination, positivity with vimentin, synaptophysin, CD56, neuron-specific enolase, and S-100 was found. On the magnetic resonance imaging, it was reported as an irregular bordered lesion of $31 \times 21 \mathrm{~mm}$ on the left side of bladder posterolateral wall.
Laparoscopic partial cystectomy was performed with synchronous transurethral view in lithotomic position (Figure 2). The BP of the patient, whose intra-operative BP measurements increased up to $200 / 140 \mathrm{~mm} \mathrm{Hg}$, was kept under control by antihypertensive medication. The final pathology was reported as paraganglioma. No primary tumorwasfound elsewhere. Postoperative 4-year follow-up showed no tumor recurrence or metastasis and no newsymptoms.

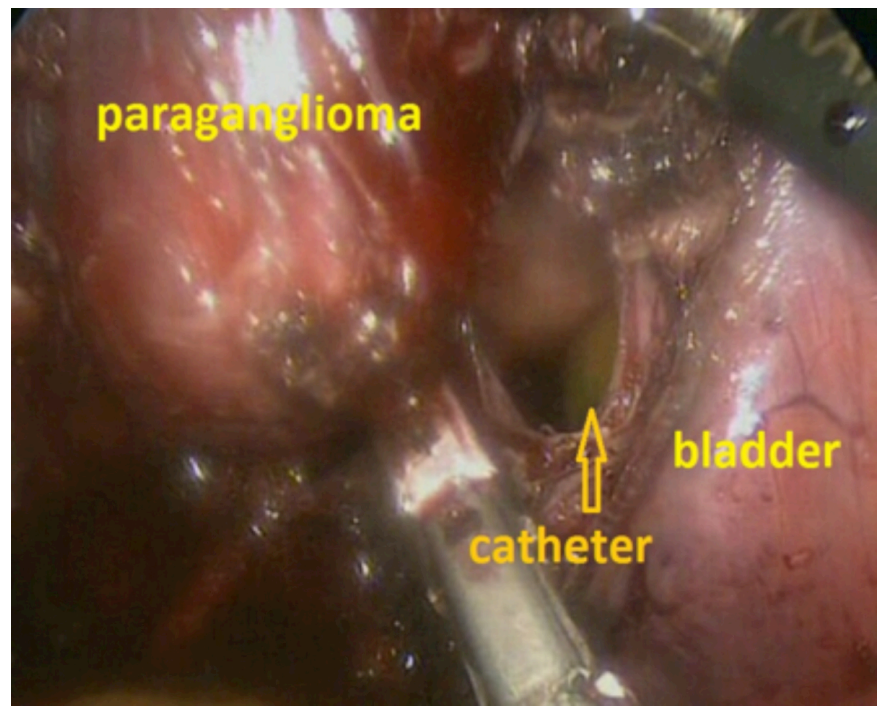

Figure 2: Laparoscopic view of retrovesical mass.

Paragangliomas are rare tumors originating from neuroendocrine cells associated with sympathetic nervous system. Tumors formed by these neuroendocrine cells originating from adrenal medulla are termed pheochromocytomas. Approximately $10 \%$ of the pheochromocytomas are extra-adrenal and termed as paragangliomas. ${ }^{1,2}$ These tumors can be found in practically every site along the migration route of the neural cells. ${ }^{2}$ Bladder paragangliomas comprise $6 \%$ of all extra-adrenal paragangliomas. These are quite rare, and comprise $0.06 \%$ of all bladder tumors. The majority of genitourinary paragangliomas originate from the bladder (79.2\%). The bladder is followed by urethra $(12.7 \%)$, renal pelvis $(4.9 \%)$ and ureter (3.2\%). ${ }^{3}$ According totheir clinical characteristics, theseareclassified into functional and non-functional types ${ }^{4}$. Functional type is seen with $83 \%$ frequency in about 200 cases in the literature. ${ }^{5}$ Non-functional type is rarer and is difficult to diagnose preoperatively; and may be more dangerous/problematic due to intraoperative unexpected hypertensive attack risk. ${ }^{4,5}$ In our case, no typical clinical symptoms were observed, but hypertensive attack was observed during the operation/surgery. Gupta et al. ${ }^{6}$ reported that non-functional paragangliomas caused tachycardia and changes in blood pressure during the surgery.

Among the treatment options, there are transurethral resection and partial or total cystectomy including pelvic lymph node dissection, if necessary. Although gold standard method is controversial, laparoscopic partial cystectomy could be used as first-line procedure in pre-operatively diagnosed bladder para- 
ganglioma cases, as it is less invasive and shows speedy recovery. ${ }^{2,7}$ There is no consensus on the follow-up procedure. Al-Zahrani et al. reported that blood and urine parameters, cystoscopy and imaging methods should be performed at least once a year. $^{5}$

Simultaneous use of transurethral and laparoscopic extravesical approach seems be a more reliable option for the excision of this rare lesion originating from the urinary bladder wall and growing into the retrovesical area. We believe that this approach may reduce intraoperative complications and increase the probability of negative surgical margins.

\section{CONFLICT OF INTEREST:}

The authors have no conflict of interest.

\section{PATIENT'S CONSENT:}

A written informed consent was obtained from the patient for the publication and the use of accompanying images of this case report.

\section{AUTHORS' CONTRIBUTION:}

MCC, NK, LS: Project development, data collection, analysis, manuscript writing.

\section{REFERENCES}

1. Pahwa HS, Kumar A, Srivastava R, Misra S, Goel MM. Urinary bladder paraganglioma: A case series with proposed treating algorithm based on our experience and review of literature. Indian J Surg Oncol 2013; 4(3):294-7. doi: 10.1007/s13193-013-0244-9.

2. Shindorf ML, Chaudhuri PK. Single-agent thalidomide for treatment of malignant paraganglioma of the organ of zuckerkandl. Case Rep Med 2019; 2019:7185973. doi: 10.1155/2019/7185973.

3. Hanji AM, Rohan VS, Patel JJ, Tankshali RA. Pheochromo- cytoma of the urinary bladder: A rare cause of severe hypertension. Saudi J Kidney Dis Transpl 2012; 23(4): 813-6. doi: 10.4103/1319-2442.98167.

4. Zhai H, Ma X, Nie W, Li H, Peng C, Li X, et al. Paraganglioma of the urinary bladder: A series of 22 cases in a single center. Clin Genitourin Cancer 2017; 15(5):765-71. doi: 10.1016/j.clgc.2017.03.010.

5. Al-Zahrani AA. Recurrent urinary bladder paraganglioma. Adv Urol 2010; 2010:912125. doi: 10.1155/2010/912125.

6. Gupta V, Sharma J, Sangwaiya A, Kaira V, Samal S, Sen R. Non-functioning paraganglioma of the urinary bladder: $A$ rare entity. Clin Cancer Investig J 2015; 4(2):268-70.

7. Lei $Y$, Tong S, Zu X, Li Y, He W, Hu X, et al. Extraperitoneal and transperitoneal laparoscopic partial cystectomy for benign non-urothelial bladder tumors: an initial experience. Urol Int 2015; 94(2):149-55. doi: 10.1159/ 000366067.

Mehmet Caglar Cakici ${ }^{1}$, Nihat Karakoyunlu ${ }^{2}$ and Levent Sagnak ${ }^{1}$

${ }^{1}$ Department of Urology, Istanbul Medeniyet University, Goztepe Training and Research Hospital, Istanbul, Turkey ${ }^{2}$ Department of Urology, Health Sciences University, Diskapi Yildirim Beyazit Education and Research Hospital, Ankara, Turkey

Correspondence to: Dr. Mehmet Caglar Cakici, Department of Urology, Istanbul Medeniyet University Goztepe Training and Research Hospital, Istanbul, Turkey

E-mail: mcaglarcakici@hotmail.com

Received: August 26, 2019; Revised: November 13, 2019; Accepted: November 13, 2019

DOI: https://doi.org/10.29271/jcpsp.2020.10.1113 